Manuscript Number: EPSR-D-19-01851R1

Title: Power Factor Improvement by Active Distribution Networks During Voltage Emergency Situations

Article Type: VSI:PSCC 2020

Keywords: Active distribution networks; distributed generation; emergency control; TSO-DSO interaction; voltage stability.

Corresponding Author: Mr. Luis David Pabon Ospina,

Corresponding Author's Institution: Fraunhofer IEE

First Author: Luis David Pabon Ospina

Order of Authors: Luis David Pabon Ospina; Thierry Van Cutsem

Manuscript Region of Origin: GERMANY

Abstract: As more Distributed Generation Units (DGUs) are integrated into medium-voltage grids, the interaction between transmission and distribution networks plays a higher role in power system security. The contribution of the so-called Active Distribution Networks (ADNs) to system stability is expected to become more and more significant. This paper focuses on long-term voltage stability issues and shows that, contrary to what might be expected, a mere reactive power injection by DGUs has detrimental effects on system stability and might bring along counterproductive effects that precipitate voltage instability and collapse. Hence, an alternative control scheme for power factor improvement by ADNs during voltage emergency situations is proposed. The power factor is measured at the point of common coupling between transmission and distribution networks. The controller enhances system stability and eliminates the identified counterproductive effects. The conclusions are derived from time domain simulations of a very simple, but concept-friendly, 5-bus system. 


\section{Power Factor Improvement by Active Distribution Networks During Voltage Emergency Situations}

\author{
Luis David Pabón Ospina \\ Dept. of System Stability and Grid Integration \\ Fraunhofer IEE, Kassel, Germany \\ luis.david.pabon.ospina@iee.fraunhofer.de
}

\author{
Thierry Van Cutsem \\ Fund for Scientific Research (FNRS) \\ University of Liège, Belgium \\ t.vancutsem@uliege.be
}

\begin{abstract}
As more Distributed Generation Units (DGUs) are integrated into medium-voltage grids, the interaction between transmission and distribution networks plays a higher role in power system security. The contribution of the so-called Active Distribution Networks (ADNs) to system stability is expected to become more and more significant. This paper focuses on longterm voltage stability issues and shows that, contrary to what might be expected, a mere reactive power injection by DGUs has detrimental effects on system stability and might bring along counterproductive effects that precipitate voltage instability and collapse. Hence, an alternative control scheme for power factor improvement by ADNs during voltage emergency situations is proposed. The power factor is measured at the point of common coupling between transmission and distribution networks. The controller enhances system stability and eliminates the identified counterproductive effects. The conclusions are derived from timedomain simulations of a very simple, but concept-friendly, 5-bus system.
\end{abstract}

Index Terms-Active distribution networks, distributed generation, emergency control, TSO-DSO interaction, voltage stability.

\section{INTRODUCTION}

Voltage instability results from the inability of the combined transmission and generation system to provide the power requested by loads [1]. In traditional power systems, i.e. with passive Distribution Networks (DNs), system operators have limited controlability over the requested power consumption (leaving aside demand response efforts). Nowadays, the increasing penetration of Distributed Generation Units (DGUs) at medium-voltage level, combined with their relatively high controlability, makes them an attractive solution to manipulate the power requested by the DNs during emergencies.

To illustrate the idea, let us consider the simple system in Fig. 1. It can be derived that, the feasible region of the power space, i.e. the set of the $\left(P_{t}, Q_{t}\right)$ pairs that the combined transmission-generation system can supply to the load, corresponds to the area below the parabola in Fig. 1. This shows that, in theory, any $P_{t}$ can be supplied, provided that the reactive counterpart $Q_{t}$ is adjusted [1]. This makes even more attractive the Active Distribution Network (ADN) power factor improvement during emergencies. If the DGUs can adjust the requested reactive power (by modifying $Q_{g}$ ), the requested $P_{t}$ can be supplied with lower risk of voltage instability. Nevertheless, there are two main problems with this approach:

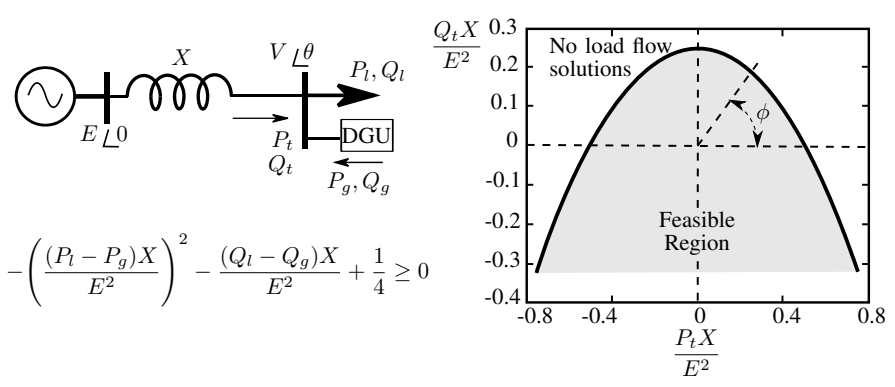

Fig. 1. A simple test system and its load power space.

1) if the DGUs increase their reactive power productions, the DN voltages can reach unacceptable high values, and 2) due to the high DN voltages, the voltage sensitive load consumption is increased. This results in a counterproductive effect that endangers voltage stability [2], [3].

The proposed controller is based on the synchronization of the DGUs and the Load Tap Changer (LTC) of the corresponding distribution transformer in order to improve the power factor as seen by the transmission network, while still keeping distribution voltages depressed. This reduces the risk of the above mentioned counterproductive effects.

The rest of this paper is organized as follows. The test system is detailed in Section II. Section III reviews the instability mechanism and the problem definition. The proposed control scheme is explained in Section IV. The simulation results are presented in Section $\mathrm{V}$ and the most significant points of this paper are summarized in Section VI.

\section{TEST SYSTEM}

The conclusions derived in this paper are based on the simple, but concept-friendly 5-bus system depicted in Fig. 2. It consists of a combined transmission-distribution system inspired by [1], in which it is used to illustrate the long-term voltage instability phenomenon. On the transmission side, a network represented by a Thévenin equivalent feeds the load center through two long transmission lines. A synchronous generator equipped with Automatic Voltage Regulator (AVR) and OverExcitation Limiter (OEL) as detailed in [4] and implemented in [5] is also considered. The transmission and distribution systems are connected through an LTC-equipped 


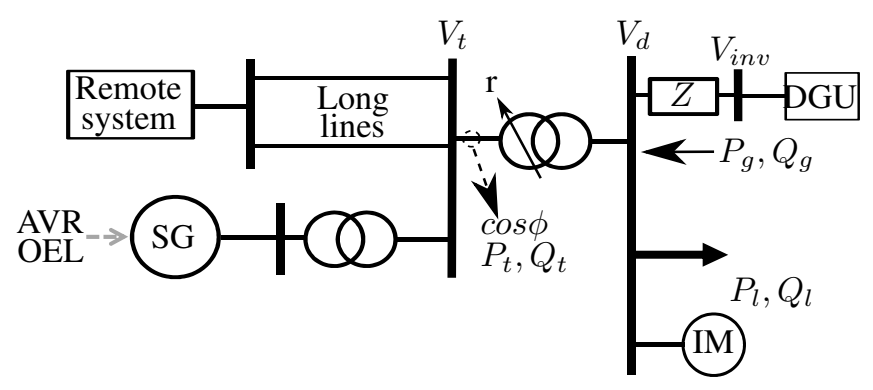

Fig. 2. 5-bus system.

transformer, whose high-voltage side corresponds to the TSODSO point of common coupling. At this point, power factor, active and reactive powers as seen by the transmission system are monitored. The transformer controls the distribution voltage $V_{d}$ between 0.99 and $1.01 \mathrm{pu}$.

The ADN consists of an exponential load, a dynamic load and a DGU.

The exponential load represents $70 \%$ of the total consumption and is modeled according to:

$$
\begin{aligned}
& P=P_{0}\left(\frac{V}{V_{0}}\right)^{\alpha} \\
& Q=Q_{0}\left(\frac{V}{V_{0}}\right)^{\beta}
\end{aligned}
$$

where, $P_{0}, Q_{0}$ and $V_{0}$ are the initial values of the active power, reactive power and terminal voltage, respectively. The exponents are initially set to $\alpha=1$ (constant current) and $\beta=2$ (constant impedance). By modifying these exponents, different voltage sensitivities of load can be obtained.

The dynamic load consists of an induction motor which represents $30 \%$ of the total active power consumption. The equivalent motor is representative of small residential and industrial motors [6]. Constant mechanical torque is considered and the power factor is improved by means of a parallel shunt capacitor.

As for the DGU, it supplies $25 \%$ of the total load. A simplified version of the WECC model for inverter-based generators (IBGs) is used. The model is detailed in [7] and its implementation can be found in [8]. The simplification consists of omitting: 1) all dynamic components not strictly necessary for long-term voltage stability analysis (e.g. the fast reactive current injection during system faults), and 2) all components characteristic of large-scale utilities (such as the plant-level controller). The model is set to local active and reactive power control as it is common practice in distribution networks. Active current is prioritized and the maximum current magnitude is set to $1.1 \mathrm{pu}$ on the DGU converter base.
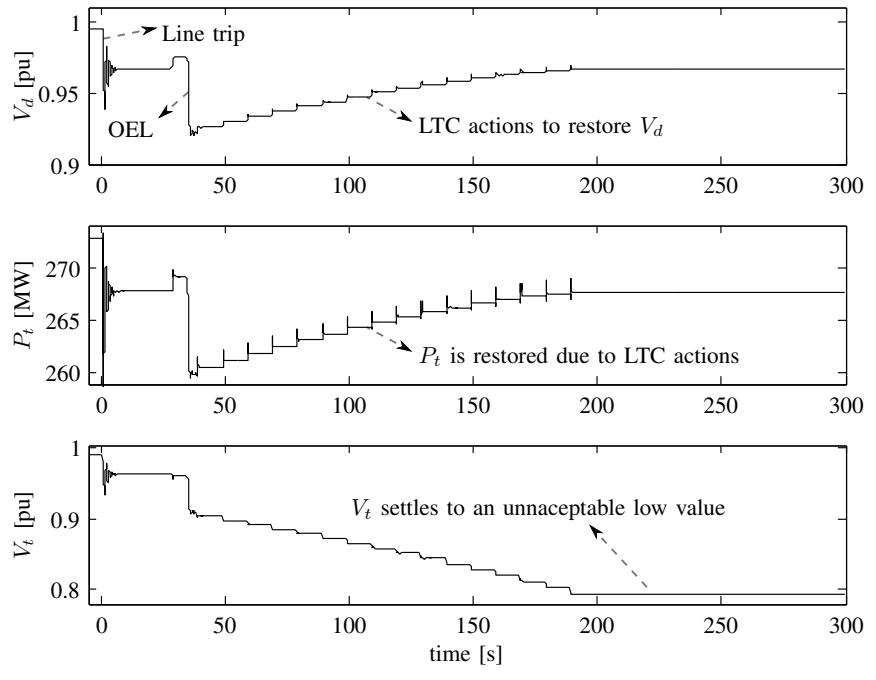

Fig. 3. Instability mechanism.

\section{INSTABILITY MECHANISM AND PROBLEM DEFINITION}

\section{A. Instability mechanism}

Voltage instability is a load-driven phenomenon usually initiated by a weakening of the transmission capability after a system disturbance such as the tripping of a transmission line.

As long-term voltage instability is dynamic by nature and emergency actions are triggered by the time evolution of the system, time-domain simulation is employed in this work.

To exemplify the phenomenon, let us assume the tripping of one of the long transmission lines in Fig. 2. The voltage evolution at both transmission and distribution levels is shown in Fig. 3. The active power consumption seen by the transmission system is depicted as well. From here on, this case in which there is no control action by the ADN, will be referred to as Case 0.

The chain of events runs as follows. The transmission line trips at $t=0$, the system shows damped oscillations and settles to a new equilibrium after a few seconds, when the oscillations have died out. In this new equilibrium, the transmission voltage settles to $V_{t}=0.963 \mathrm{pu}$. Thus, the system is short-term stable. However, this operating point does not correspond to a long-term equilibrium. At $t=29 \mathrm{~s}$, the LTC of the distribution transformer starts decreasing the ratio $r$ in its attempt to restore the distribution voltage. As a consequence, it starts restoring the power consumption, as seen in Fig. 3. At $t=36 \mathrm{~s}$, however, the OEL of the synchronous generator operates, reducing and limiting the field current to avoid generator damage. At this point, the generator loses its voltage control capability. The LTC continues lowering the transformer ratio in unsuccessful attempts to bring the distribution voltage inside the normal operation deadband. As a consequence, $P_{t}$ is further (but partly) restored, depressing even more the transmission voltage. The process continues until the LTC hits its limit and $V_{t}$ settles to an unacceptable low value of $0.79 \mathrm{pu}$. This is considered as system instability even 
though the short-term dynamics remain stable (the generator keeps synchronism) [1].

\section{B. Problem definition}

A classical technique to counteract voltage instability consists in lowering the LTC voltage setpoint of distribution transformers (e.g. to $0.95 \mathrm{pu}$ ). This corrective action exploits the voltage sensitivity of loads. The power consumption is decreased thanks to the intentionally reduced distribution voltage, thus, decreasing the system stress during the emergency situation. This technique has proven to be effective in passive distribution networks.

With the increased number of DGUs in medium-voltage networks, there have been recent efforts aiming to improve long-term voltage stability using the assets of ADNs. For instance, [2] and [3] explore the possibility of DGUs injecting reactive power after a voltage instability alarm is received. At first sight, this seems to be a proper course of action under a situation of potential voltage instability. Even knowing the resistive nature of DNs, if voltage is falling, it seems natural to support it by means of reactive power injection nearby the load centers. Nevertheless, as mentioned in the Introduction, reactive power injection actually brings along counterproductive effects.

Indeed, when reactive power is injected by the DGUs, the load consumption is increased as well, due to the increased load-side voltage. Then, stability becomes a trade-off between the benefits of injecting reactive power, and its counterproductive effects on load power restoration. If the reactive power support is not enough to counteract the load power restoration that it brings along, an attempt is made to bring the total consumption seen by the transmission system outside the parabola in Fig. 1 and system instability will result. This is the main counterproductive effect that endangers system stability if the DGUs inject reactive power without being properly synchronized with the LTC [2].

To avoid this undesired effect, references [2] and [3] adopted the opposite strategy of decreasing the DGU reactive power injections with the aim of reducing the load-side voltage, and therefore, the consumption of voltage-sensitive loads. This approach has proven to be successful in the scenarios detailed in [2], [3]. Nevertheless, there is one drawback: the reactive power support of DGUs cannot be exploited. In fact, the DGUs decrease and may even consume reactive power during the emergency situation.

To resolve this dilemma, this paper proposes a method to exploit the reactive power support by DGUs while controlling the LTC to avoid load power restoration.

\section{THE PROPOSED CONTROL SCHEME}

In order to facilitate its implementation, the controller reuses the control logic of traditional LTCs as follows. The LTC adjusts the transformer ratio in order to keep the distribution voltage inside a pre-defined deadband. It has intentional delays. When the distribution voltage leaves its deadband at time $t_{0}$, the first tap change takes place at time $t_{0}+\tau_{1}$ and the subsequent changes at times $t_{0}+\tau_{1}+k \tau_{2}(\mathrm{k}=1,2, \ldots)$ The delay is reset to $\tau_{1}$ after the controlled voltage has re-entered (or jumped from one side to the other of) the deadband [4]. Note that the control scheme can also be implemented on LTCs with inverse time characteristics instead of constant delays.

The control scheme is based on the synchronization of LTC actions and DGU reactive power injections as shown in Fig. 4 with a finite state machine formulation. The decisions are based on two parameters:

- $V_{d}^{L T C}$ : Voltage setpoint for the LTC during the emergency. As a traditional countermeasure, this setpoint is lowered to a pre-defined value, e.g. $0.95 \mathrm{pu}$ to exploit the voltage sensitivity of loads while staying in a reasonable range of load voltages [9], [10].

- $V_{d}^{D G U}$ : Value of $V_{d}$ at which the DGUs stop increasing the reactive power injection. This allows avoiding the undesired power restoration.

Both parameters $V_{d}^{L T C}$ and $V_{d}^{D G U}$ have associated deadbands, $d b_{L T C}$ and $d b_{D G U}$, respectively.

The only two necessary control actions are described as follows:

$$
L T C_{\text {action }}: \begin{cases}\text { tap }:=\operatorname{tap}-1 & \text { if } V_{d}<V_{d}^{L T C}-d b_{L T C} \\ \text { tap }:=t a p+1 & \text { if } V_{d}>V_{d}^{L T C}+d b_{L T C} \\ \text { tap unchanged } & \text { otherwise }\end{cases}
$$

$$
D G U_{\text {action }}: \begin{cases}Q_{\text {increase }} & \text { if } V_{d}<V_{d}^{D G U}-d b_{D G U} \\ Q_{\text {decrease }} & \text { if } V_{d}>V_{d}^{D G U}+d b_{D G U} \\ Q \text { unchanged } & \text { otherwise }\end{cases}
$$

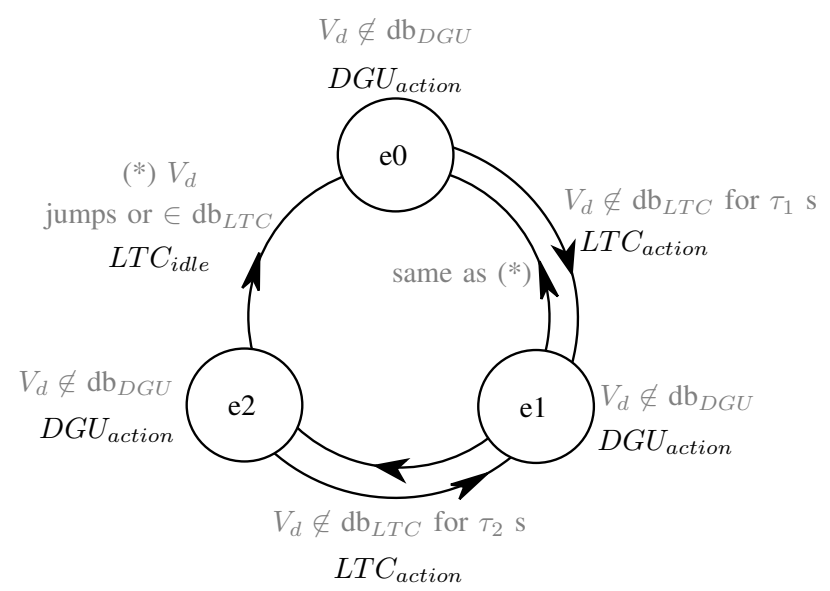

Fig. 4. Control scheme - actions are shown in black, while conditions that trigger state transitions and control actions are shown in grey.

The controller step-by-step operation is going to be explained using the results in Sections V-A and V-B. 


\section{Simulation RESUlts}

Two more cases are investigated to analyze the controller performance (see Table I). The following parameters are used: $V_{d}^{L T C}$ is set to $0.95 \mathrm{pu}$ in all simulations. This is a typical value for conservation voltage reduction [11] and it exploits the voltage sensitivity of loads while staying in a reasonable range of load voltages. In Case 1, the parameter $V_{d}^{D G U}$ is set to the same value of $V_{d}^{L T C}(0.95 \mathrm{pu})$. Subsequently, $V_{d}^{D G U}$ is set to $0.975 \mathrm{pu}$ in Case 2, i.e. slightly higher than $V_{d}^{L T C}$. As for the deadbands, it is a common practice in LTC controllers to have a total deadband $\left( \pm d b_{L T C}\right)$ of at least twice the voltage variation from one tap position to the next. This ensures that $V_{d}$ does not jump from one side of the deadband to the other with a single tap operation. In this case, the LTC adjusts the transformer ratio in the range [0.88 - 1.20] over 33 positions (thus from one position to the next, the ratio varies by 0.01 ). Therefore, the value for $d b_{L T C}$ is set to $0.01 \mathrm{pu}$. The value of $d b_{D G U}$ is also set to $0.01 \mathrm{pu}$. This avoids jumping from one side of the deadband to the other after fast DGU actions. Even though DGUs allow very fast control, the DGU reactive power increase rate is intentionally limited to avoid abrupt changes. A conservative rate of $0.01 \mathrm{pu} / \mathrm{s}$ on the DGU MVA base is considered. The results are shown from Fig. 5 to Fig. 9.

TABLE I

STUDY CASES

\begin{tabular}{ccl}
\hline Case & Controller & \multicolumn{1}{c}{ Description } \\
\hline 0 & No & No emergency action \\
1 & Yes & $V_{d}^{L T C}=0.95$ pu and $V_{d}^{D G U}=0.95 \mathrm{pu}$ \\
2 & Yes & $V_{d}^{L T C}=0.95 \mathrm{pu}$ and $V_{d}^{D G U}=0.975 \mathrm{pu}$ \\
\hline
\end{tabular}

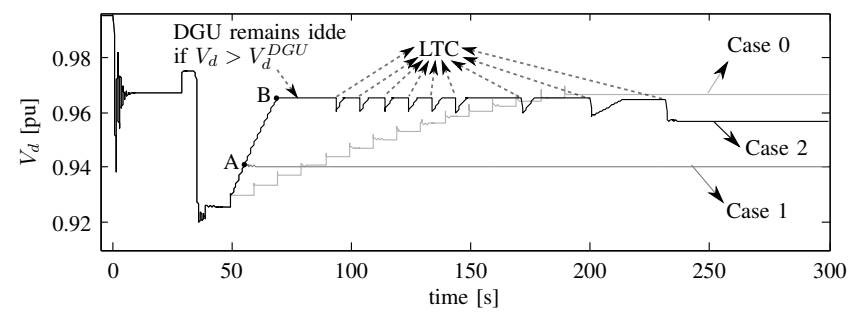

Fig. 5. Distribution voltage.

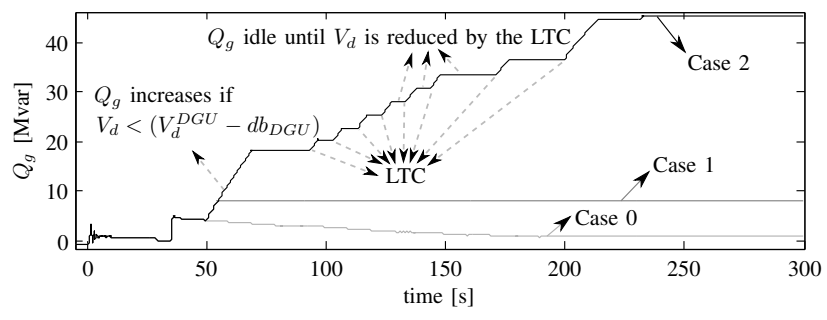

Fig. 6. DGU reactive power.

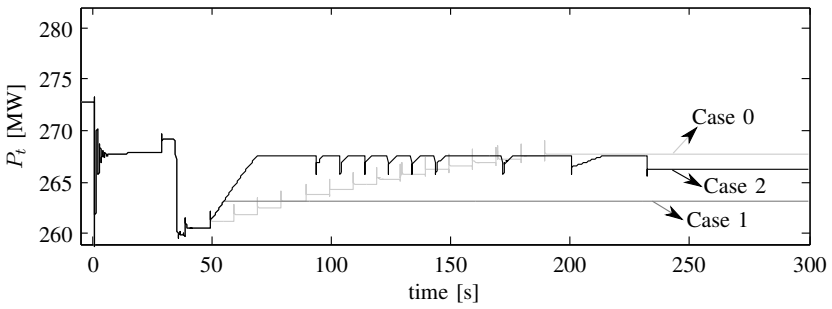

Fig. 7. Total active power consumption.

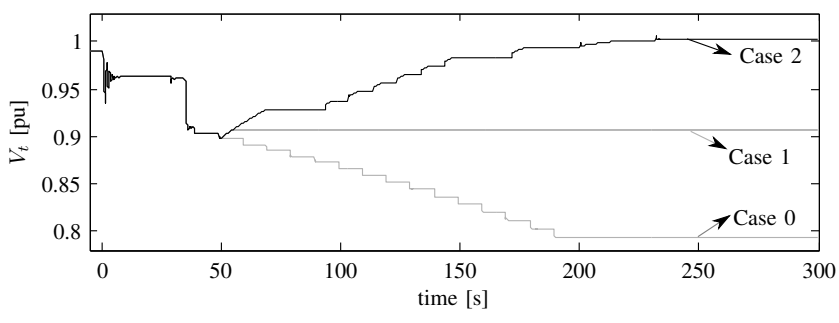

Fig. 8. Transmission voltage.

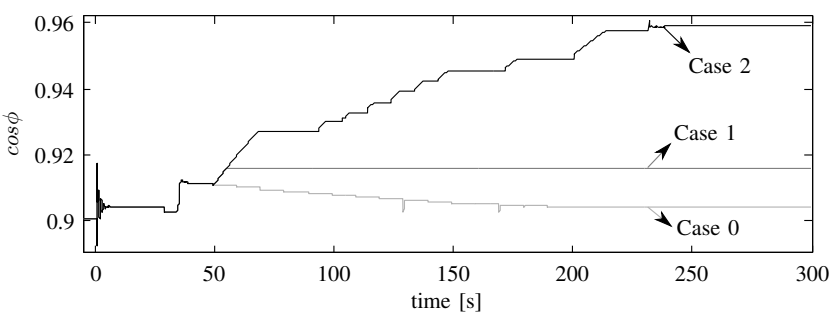

Fig. 9. Power Factor at TSO-DSO point of common coupling.

\section{A. Controller performance with $V_{d}^{D G U}=V_{d}^{L T C}=0.95 \mathrm{pu}$}

Let us start with the evolution of the distribution voltage and the DGU reactive power injection (Figs. 5 and 6 for Case 1). The chain of events runs as follows:

- The system disturbance (tripping of one of the long transmission lines) takes place at $t=0$. The LTC starts restoring power consumption in its attempt to increase $V_{d}$.

- As a consequence, the OEL of the synchronous generator operates at around $t=36 \mathrm{~s}$. The alarm is triggered at $t=50 \mathrm{~s}$, activating the emergency controller. The Local Identification of Voltage Emergency Situations (LIVES) method is used for instability detection [12]. As a common practice, a transmission alarm that is triggered when $V_{t}$ falls below a threshold ( $0.9 \mathrm{pu}$ in this case) is used as a backup.

- At this point, $V_{d}=0.926 \mathrm{pu}$ and the controller operates in state $\mathrm{e} 0$ in Fig. 4. With such a low voltage, the DGU control action in e0 will take place. According to Eq. 4, $V_{d}<V_{d}^{D G U}-d b_{D G U}$ (in this case $0.926 \mathrm{pu}<0.95-$ 0.01 ), the corresponding control action is to increase DGU reactive power. The reactive power injection starts at around $t=50 \mathrm{~s}$ as seen in Fig. 6.

- As a consequence, $V_{d}$ increases up to $0.94 \mathrm{pu}$ at $t=55 \mathrm{~s}$, when it reaches the value $V_{d}^{D G U}-d b_{D G U}$ 
(see point $A$ in Fig. 5). The third condition in Eq. 4 is met, and the DGU remains idle.

- Thanks to the fast reaction of the DGU, the condition for the transition from e 0 to e 1 was not met and the controller remains in $\mathrm{e} 0$.

- Due to the fact that the DGU quickly brought $V_{d}$ to the LTC target voltage, there is no further tap operation. The DGU and the LTC remain idle for the rest of the simulation.

This has a significant advantage. The controller stopped the instability mechanism which was caused by the LTC actions. Therefore the power restoration is avoided, as shown in Fig. 7 - Case 1, and the fall of the transmission voltage is stopped, as confirmed by Fig. 8 - Case 1. Nevertheless, there is a disadvantage with this approach. Due to the fact that $V_{d}$ settles at its maximum threshold defined by $V_{d}^{D G U}$, the DGU reactive power support is stopped at $t=55 \mathrm{~s}$ and therefore there is no further power factor improvement, see Fig. 9 - Case 1.

\section{B. Controller performance with $V_{d}^{D G U}>V_{d}^{L T C}$}

In this case, $V_{d}^{D G U}$ is set to $0.975 \mathrm{pu}$, a slightly higher value than $V_{d}^{L T C}$ which remains at $0.95 \mathrm{pu}$. To understand the controller operation, let us start with the evolution of the distribution voltage and the DGU reactive power injection (see Figs. 5 and 6 - Case 2). The new chain of events runs as follows:

- The same system disturbance takes place at $t=0$. The LTC starts restoring power consumption in its attempt to increase $V_{d}$.

- As a consequence, the OEL of the synchronous generator operates at around $t=36 \mathrm{~s}$. The same alarm as in Case 1 is triggered at $t=50 \mathrm{~s}$, activating the emergency controller.

- At this point, $V_{d}=0.926 \mathrm{pu}$ and the controller operates in state e0 in Fig. 4. As in the previous case, the corresponding control action is to increase the DGU reactive power as seen in Fig. 6 at around $t=50 \mathrm{~s}$.

- $V_{d}$ increases up to $V_{d}^{D G U}-d b_{D G U}=0.965 \mathrm{pu}$, which is reached at $t=70 \mathrm{~s}$ (see point $\mathrm{B}$ in Fig. 5). The third condition in Eq. 4 is met and the DGU remains idle. The controller is still operating in $\mathrm{e} 0$, but now $V_{d}=0.965 \mathrm{pu}$ and the DGU is idle.

- The value of $V_{d}$ is above the deadband of the LTC $\left(V_{d}^{L T C}+d b_{L T C}=0.95+0.01=0.96 \mathrm{pu}\right)$. The condition for the transition from $\mathrm{e} 0$ to $\mathrm{e} 1$ is met at $t=94 \mathrm{~s}$. The corresponding control action is to increase the tap position according to Eq. 3. This decreases $V_{d}$ to $0.96 \mathrm{pu}$ as seen in Fig. 5 - Case 2 shortly after $t=94 \mathrm{~s}$.

- The controller operates in e1, with $V_{d}=0.96 \mathrm{pu}$. The condition for the DGU control action in e1 is now met again, due to the fact that $V_{d}<V_{d}^{D G U}-d b_{D G U}$. Therefore, the DGU injects some more reactive power starting at $t=94 \mathrm{~s}$ as seen in Fig. 6 .

- Just a few seconds later, this new reactive power injection makes $V_{d}$ reach 0.965 pu once again, putting the DGU to idle.
- The controller operates only a few more seconds in e1 with idle DGU and $V_{d}=0.965 \mathrm{pu}$, until the condition for transition from e1 to e 2 is met at $t=104 \mathrm{~s}$. The tap position is increased again according to Eq. 3, reducing $V_{d}$ to $0.96 \mathrm{pu}$.

- The controller falls into e2, $V_{d}=0.96 \mathrm{pu}$, so the condition for DGU reactive power increase is met again.

- The controller iterates a few more times, synchronizing LTC actions and DGU reactive power injections.

This proper synchronization allows the DGU to support reactive power without increasing $V_{d}$ above $V_{d}^{D G U}$. As a consequence, the undesired active power restoration due to reactive power support is not an issue anymore (see Fig. 7 Case 2). On the other hand, the power factor measured at the TSO-DSO interface is highly improved (see Fig. 9) and the transmission voltage recovers even around its pre-disturbance value (see Fig. 8).

It is important to note that the controller does not iterate indefinitely. On the contrary, the process stops once the DGU reaches a pre-defined limit for maximum reactive power injection $Q_{\max }$ (set to a conservative value of $0.4 \mathrm{pu}$ on the inverter MVA base.) In any case, the DGU stops increasing its reactive power injection as soon as its current hits its maximum permissible value. This is to prevent damage in the inverter and it is part of any commercial DGU. In order to prevent overvoltages inside the $\mathrm{ADN}$, the DGU also stops the reactive power injection increase if the terminal voltage ( $V_{i n v}$ in Fig. 2) hits a predefined threshold.

At this point, the control scheme has proven its effectiveness in the studied scenarios. The next two sections explore two critical aspects for its success: a) the sensitivity of loads to voltage, and b) the reactive power reserve available in the DGU at the moment of alarm.

\section{Controller response with low voltage sensitivity of loads}

The proposed controller exploits the voltage sensitivity of loads, which is characterized by the exponents $\alpha$ and $\beta$ in Eqs. 1 and 2, for the non-motor load.

To exploit such sensitivity, the load-side voltage $V_{d}$ is reduced during the emergency. For instance, if $V_{d}$ is reduced by $5 \%$, the load active power is expected to decrease by $5 \%$, and the reactive power by approximately $10 \%$. The same technique is used to reduce peak demands and energy consumption, and is known as conservation voltage reduction [11]. The induction motor, on the other hand, consumes an almost constant active power irrespective of the voltage.

Thus, the sensitivity of loads toward voltage variations is critical for the success of the proposed control scheme. In order to analyze the controller performance in DNs with lower voltage sensitivity of the non-motor loads, a more severe case is presented in Figs. 10 to 14. The exponents $\alpha$ and $\beta$ have been decreased to 0.8 and 1.0 , respectively. Note that the presence of the motor load yields an effective value of $\alpha$ lower than 0.8 for the composite load. 


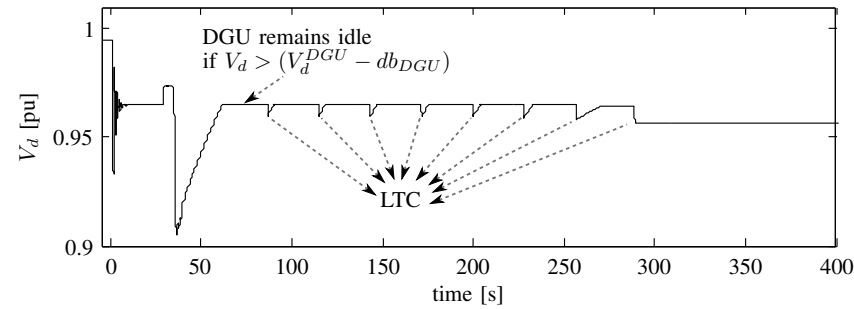

Fig. 10. Distribution voltage with low load sensitivity to voltage.

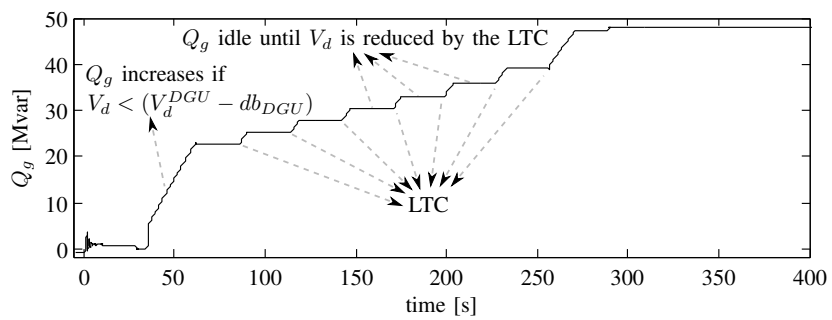

Fig. 11. DGU reactive power with low load sensitivity to voltage.

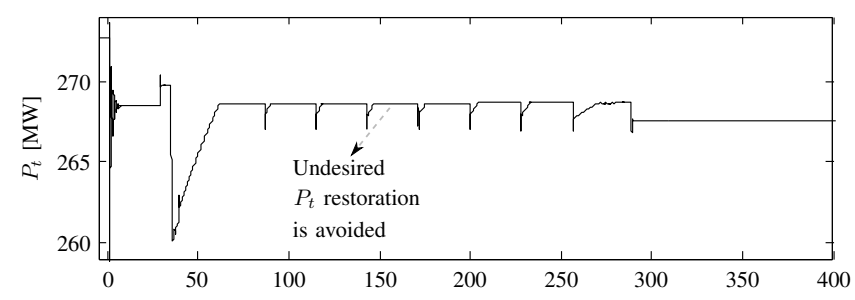

Fig. 12. Total active power consumption with low load sensitivity to voltage.

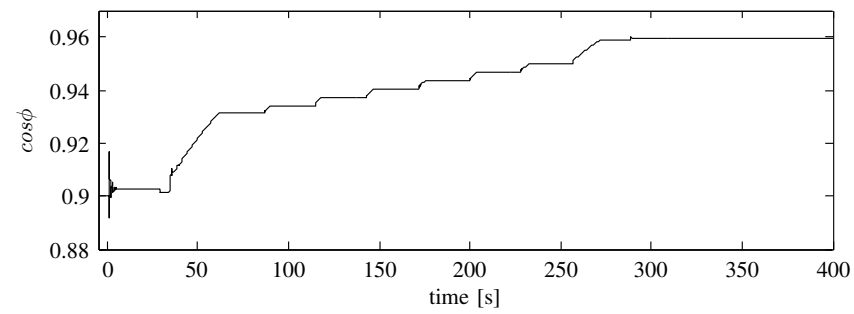

Fig. 13. Power Factor with low load sensitivity to voltage.

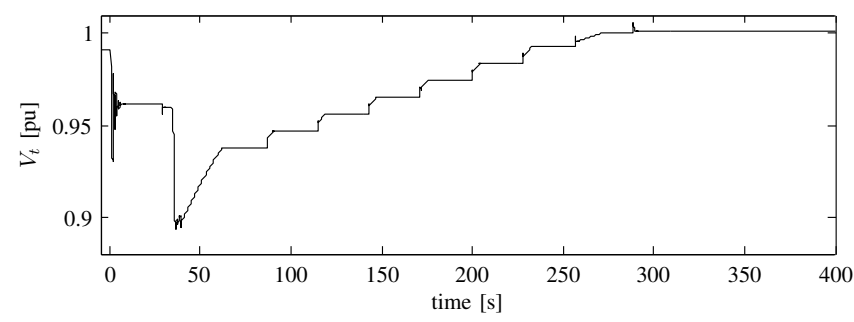

Fig. 14. Transmission voltage with low load sensitivity to voltage.

Although it takes around $59 \mathrm{~s}$ more than in Case 2 for $V_{t}$ to reach a new long-term equilibrium, the controller proves to be also effective in this case.

Thanks to the controller action, all system variables, including transmission and distribution voltages, settle to acceptable values. The power factor has been again improved to 0.96 (see Fig. 13), while the total active power consumption seen by the transmission system has not been restored. This supports the transmission voltage which reaches $V_{t}=1.001 \mathrm{pu}$ in the long term (see Fig. 14).

\section{Impact of $D G U$ reactive power reserve}

The proposed control scheme relies on the ability of DGUs to increase reactive power production when requested. Therefore, the reactive power available in the ADN at the moment of alarm has a significant impact on its success.

Given a certain penetration level of DGUs, this reactive power reserve depends on two factors: the capability curve of the DGUs, and their operating point at the moment of alarm. To exemplify this, consider the typical capability curve shown in Fig. 15 (in this case, given in terms of inverter current). Modern inverters can operate at any point of the semi-circle, i.e. the inverter can provide any pair of reactive and active currents $\left(i_{Q}, i_{P}\right)$, as long as the current magnitude remains below its maximum limit $i_{\max }$ (typically in the range of 1.1 to $1.3 \mathrm{pu}$ ). In order to be conservative, a maximum current of $1.0 \mathrm{pu}$ is considered in this section.

Let us first assume that the DGU initial operating point is $i_{P}=i_{P 01}$ and $i_{Q}=0$ as shown in Fig. 15. In this scenario, if the emergency control requests a reactive power injection, the DGU can increase its reactive current $i_{Q}$ until the maximum magnitude $i_{\max }$ is reached. This gives the reactive current margin $i_{Q \operatorname{margin} 1}$. On the other hand, if the DGU initial operating point is $\left(0, i_{P 02}\right)$, when the emergency control scheme requests a reactive power injection, the reactive current can be increased by a smaller amount $i_{Q \operatorname{margin} 2}$. Note that, besides the limit imposed by $i_{\max }$, some control schemes have an additional limit for the maximum reactive power injection $Q_{\max }$, which could be reached before the maximum current limit.

In order to see the impact of the reactive power reserve on the effectiveness of the proposed control scheme, four different initial values for $i_{P}$ are considered $(0.85,0.91,0.93$ and $0.96 \mathrm{pu}$ ). This gives pre-disturbance reactive power margins of $15,12,10$ and $8 \%$ of the total load consumption, respectively. The same contingency is applied and the evolution of the DGU current is shown in Fig. 16.

It should be noted that, after the contingency, the active current needs to be adjusted in order to keep the active power setpoint while the voltage decreases. This leads to an increase

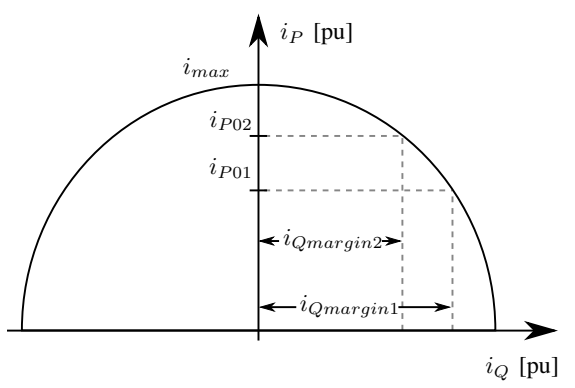

Fig. 15. DGU current capability diagram. 


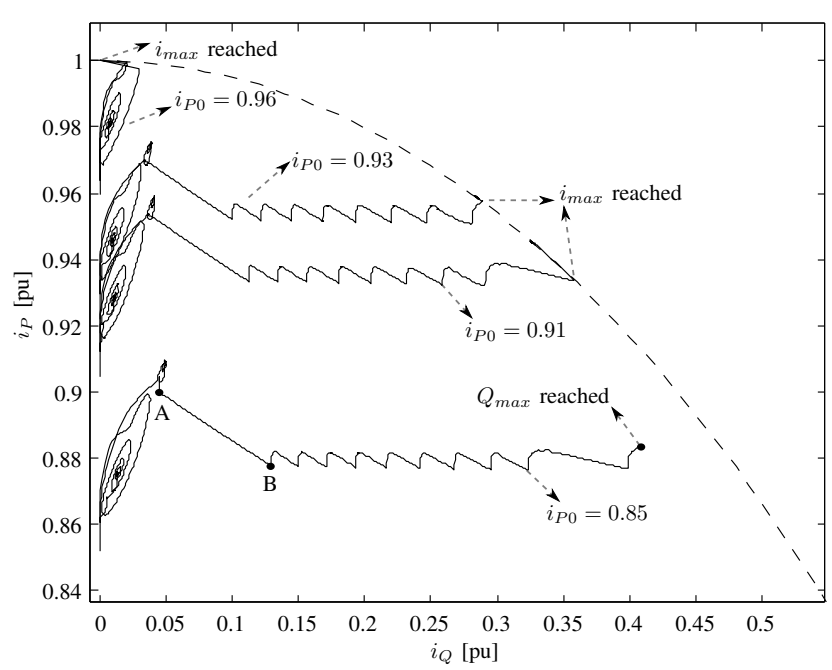

Fig. 16. Evolution of the DGU current for different initial active current values.

in active current, and the post-contingency reactive power margin is smaller compared to its pre-disturbance value.

In the first case $\left(i_{P 0}=0.85 \mathrm{pu}\right)$, the DGU current oscillates after the system contingency. The emergency control scheme is activated at point A in Fig. 16. The process of the control scheme can be seen, with the first LTC action taking place at point $\mathrm{B}$. The DGU is able to increase reactive current until the reactive power reaches its maximum value $Q_{\max }$ (in this case $0.4 \mathrm{pu}$ ). This happens before the current hits $i_{\max }$, resulting in a post-contingency reactive power margin of about $11 \%$ of the total load consumption.

In the cases with $i_{P 0}=0.91$ and $0.93 \mathrm{pu}$, the DGU increases $i_{Q}$ until $i_{\max }$ is reached. The post-contingency reactive power margin is $9 \%$ and $8 \%$ of the total load consumption, respectively. The control scheme succeeds in improving power factor above 0.94 as seen in Fig. 17 (both cases show a very similar behaviour and, for legibility reasons, only the simulation with $i_{P 0}=0.91 \mathrm{pu}$ is presented).

The result is different when the initial active current is $0.96 \mathrm{pu}$. In order to keep the DGU active power setpoint, full active current is necessary after the contingency. The initial reactive power margin is so low, that the controller has little chance to inject any reactive power when needed. In fact, after the initial oscillations have died-out, there is no reactive power margin at all. Therefore, unlike in the last three cases, the power factor cannot be improved (see Fig. 17). This is a natural limitation of the controller. Nevertheless, note that the last case is very conservative, i.e. very low initial reactive power margin and very low maximum current $i_{\max }=1.0 \mathrm{pu}$. In most cases, $i_{\max }$ will range from 1.1 to $1.3 \mathrm{pu}$, increasing the reactive power reserve. It must be also noted that, even if there is no reactive power injection, the control scheme still stops the instability mechanism in the same way as in the classical scheme that lowers the LTC setpoint. In this case, the fall of transmission voltage stops at around $t=85 \mathrm{~s}$ when it settles to $0.91 \mathrm{pu}$.
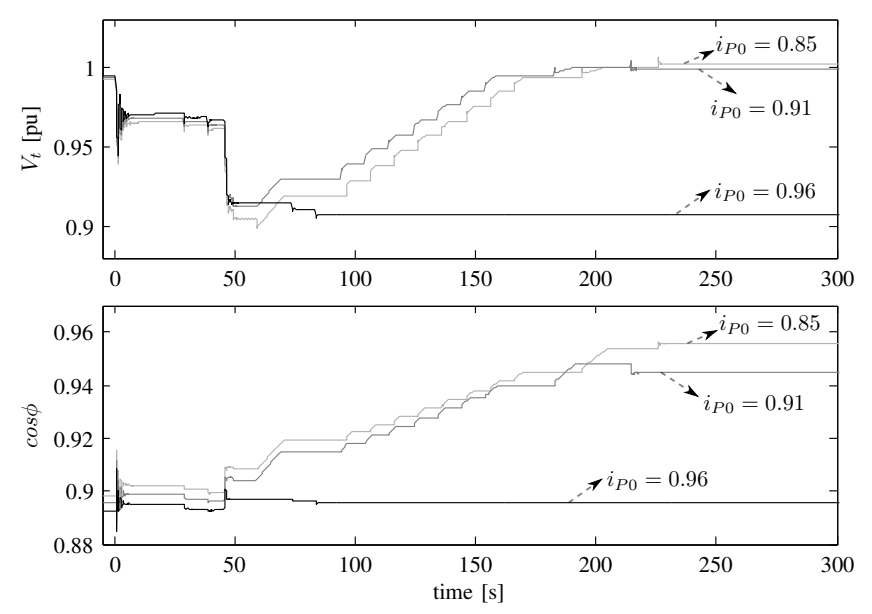

Fig. 17. Evolution of transmission voltage and power factor for different initial active current values.

\section{CONCLUSION}

As more DGUs are integrated into medium-voltage grids, the interaction between transmission and distribution networks plays a higher role in power system security. In this paper, a voltage emergency controller involving distribution network assets has been proposed and tested on a simple, but conceptfriendly 5-bus system. The controller improves the power factor seen by the transmission network during emergency situations. This is done by synchronized control actions between DGUs and the LTC of the distribution transformer. A simple three-state finite machine restitutes the standard control logic of LTCs, which facilitates the control scheme implementation. Since the variation of loads with voltage plays a central role in the proposed control, the scheme has been tested in severe cases of low sensitivity to voltage variations. It is concluded that, the controller can effectively improve the power factor and support transmission voltage during the emergency. Voltage stability is enhanced and the counterproductive effects that may precipitate instability are eliminated. The control scheme limitations regarding the reactive power reserve at the moment of alarm have been stressed. Unless the reactive power margin is very low (e.g. less than $8 \%$ of the total load consumption for the studied case), the controller can effectively improve voltage stability. Furthermore, it is concluded that, even in the above-mentioned conservative case, the instability mechanism is stopped avoiding the fall of transmission voltage to unacceptable low values.

\section{ACKNOWLEDGMENT}

We acknowledge the support of our work by the German Ministry for Economic Affairs and Energy and the Projekträger Jülich within the project Netzregelung 2.0 Regelung und Stabilität im stromrichter-dominierten Verbundnetz (FKZ 0350023A). Only the authors are responsible for the content of this publication. This paper does not necessarily reflect the consolidated opinion of the project consortium Netzregelung 2.0. 


\section{REFERENCES}

[1] T. Van Cutsem and C. Vournas., "Voltage Stability of Electric Power Systems," New York, NY, USA, 1998.

[2] P. Aristidou, G. Valverde, and T. Van Cutsem, "Contribution of distribution network control to voltage stability: A case study," IEEE Transactions on Smart Grid, vol. 8, no. 1, pp. 106-116, Jan 2017.

[3] IEEE Power System Dynamic Performance Committee, "Contribution to Bulk System Control and Stability by Distributed Energy Resources connected at Distribution Network," PES-TR22, Tech. Rep.,Aug. 2017.

[4] IEEE Task Force on Test Systems for Voltage Stability and Security Assessment, "Test Systems for Voltage Stability Analysis and Security Assessment," PES-TR19, Tech. Rep.,Aug. 2015.

[5] L. D. P. Ospina, A. F. Correa, and G. Lammert, "Implementation and validation of the nordic test system in digsilent powerfactory," in 2017 IEEE Manchester PowerTech, June 2017, pp. 1-6.

[6] IEEE Task Force on Load Representation for Dynamic Performance, "Standard load models for power flow and dynamic performance simulation," IEEE Transactions on Power Systems, vol. 10, no. 3, pp. 1302 1313, Aug 1995.

[7] Western Electricity Coordinating Council (WECC) Renewable Energy Modeling Task Force. (2014, April) WECC solar plant dynamic modeling guidelines. [Online]. Available: https://www.wecc.biz/Reliability/WECC Solar Plant Dynamic Modeling Guidelines.pdf

[8] G. Lammert, L. D. P. Ospina, P. Pourbeik, D. Fetzer, and M. Braun, "Implementation and validation of wecc generic photovoltaic system models in digsilent powerfactory," in 2016 IEEE Power and Energy Society General Meeting (PESGM), July 2016, pp. 1-5.

[9] C. Vournas and M. Karystianos, "Load tap changers in emergency and preventive voltage stability control," IEEE Transactions on Power Systems, vol. 19, no. 1, pp. 492-498, Feb 2004.

[10] C. D. Vournas, A. Metsiou, M. Kotlida, V. Nikolaidis, and M. Karystianos, "Comparison and combination of emergency control methods for voltage stability," in IEEE Power Engineering Society General Meeting, 2004., June 2004, pp. 1799-1804 Vol.2.

[11] Z. Wang and J. Wang, "Review on implementation and assessment of conservation voltage reduction," IEEE Transactions on Power Systems, vol. 29, no. 3, pp. 1306-1315, May 2014.

[12] C. D. Vournas and T. Van Cutsem, "Local identification of voltage emergency situations," IEEE Transactions on Power Systems, vol. 23, no. 3, pp. 1239-1248, Aug 2008. 


\section{Declaration of interests}

XThe authors declare that they have no known competing financial interests or personal relationships that could have appeared to influence the work reported in this paper.

$\square$ The authors declare the following financial interests/personal relationships which may be considered as potential competing interests:

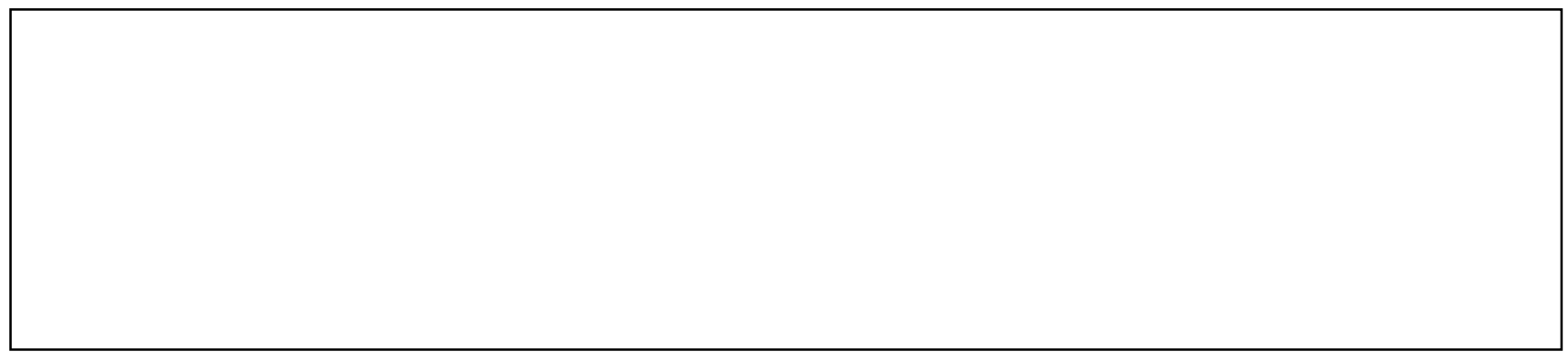

